\title{
Evaluation of variation in D-dimer levels among COVID-19 and bacterial pneumonia: a retrospective analysis
}

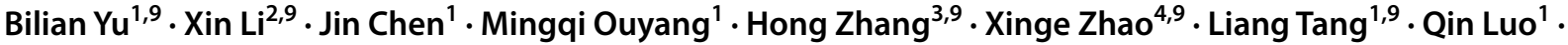 \\ Min $\mathrm{Xu}^{5,9} \cdot$ Lizhen Yang ${ }^{6,9} \cdot$ Guxiang Huang ${ }^{7,9} \cdot$ Xianling Liu $^{8,9}$. Jianjun Tang ${ }^{1,9}$
}

Published online: 10 June 2020

(c) Springer Science+Business Media, LLC, part of Springer Nature 2020

\begin{abstract}
In the recent outbreak of novel coronavirus infection worldwide, the risk of thrombosis and bleeding should be concerned. We aimed to observe the dynamic changes of D-dimer levels during disease progression to evaluate their value for thrombosis. In this study, we report the clinical and laboratory results of 57 patients with confirmed COVID-19 pneumonia and 46 patients with confirmed community-acquired bacterial pneumonia (CAP). And their concentrations of D-dimer, infection-related biomarkers, and conventional coagulation were retrospectively analyzed. The Padua prediction score is used to identify patients at high risk for venous thromboembolism (VTE). The results found that, on admission, both in COVID-19 patients and CAP patients, D-dimer levels were significantly increased, and compared with CAP patients, D-dimer levels were higher in COVID-19 patients $(\mathrm{P}<0.05)$. Besides, we found that in COVID-19 patients, D-dimer were related with markers of inflammation, especially with hsCRP $(\mathrm{R}=0.426, \mathrm{P}<0.05)$. However, there was low correlation between VTE score and D-dimer levels (Spearman's $R=0.264, P>0.05$ ) weakened the role of D-dimer in the prediction of thrombosis. After treatments, D-dimer levels decreased which was synchronous with hsCRP levels in patients with good clinical prognosis, but there were still some patients with anomalous increasing D-dimer levels after therapy. In conclusion, elevated baseline D-dimer levels are associated with inflammation but not with VTE score in COVID-19 patients, suggesting that it is unreasonable to judge whether anticoagulation is needed only according to D-dimer levels. However, the abnormal changes of D-dimer and inflammatory factors suggest that anticoagulant therapy might be needed.
\end{abstract}

Keywords D-dimer $\cdot$ COVID-19 $\cdot$ Bacterial pneumonia $\cdot$ Retrospective analysis

\section{Highlights}

- After COVID-19 outbreaks, the risk of thrombosis and bleeding has attracted much attention.

- It has been reported that abnormal D-dimer levels are associated with poor prognosis.

Bilian Yu and Xin Li make equal effort to this paper.

Electronic supplementary material The online version of this article (https://doi.org/10.1007/s11239-020-02171-y) contains supplementary material, which is available to authorized users.

Xianling Liu

liuxianling@csu.edu.cn

Jianjun Tang

tom200210@csu.edu.cn

Extended author information available on the last page of the article
- D-dimer levels were higher in COVID-19 patients and were related with markers of inflammation, and after treatments, D-dimer levels decreased which was synchronous with hsCRP levels in patients with good clinical prognosis. Also, the low correlation between Padua VTE score and D-dimer levels weakened the role of D-dimer in the prediction of thrombosis.

- The abnormal changes of D-dimer and inflammatory factors suggest that aggressive anticoagulant therapy might be needed.

\section{Introduction}

Since December 2019, a novel member of human coronavirus which newly identified in Wuhan, China, is officially named as severe acute respiratory syndrome coronavirus 2 (SARS-CoV-2) by International Committee on Taxonomy 
of Viruses, ICTV [1-3]. SARS-CoV-2, which belongs to the beta-coronavirus $2 b$ lineage in the phylogenetic tree, is a new strain of RNA viruses that has not been previously identified in humans [4]. Recently, the disease caused by SARS-CoV-2 was named as COVID-19 (coronavirus disease 2019) by World Health Organization (WHO). The numbers of infected patients worldwide increase rapidly and has exceeded 100,000 .

In previous reports [1-3], the clinical characteristics of COVID-19 patients have been investigated. Specifically, COVID-19 is usually characterized by lower respiratory tract symptoms with fever, dry cough, and dyspnea, a manifestation similar to those of two other diseases caused by coronaviruses, severe acute respiratory syndrome (SARS) and Middle East respiratory syndrome, MERS [5, 6]. The reported overall case-fatality rate (CFR) for COVID-19 by now was $2.3 \%$, but cases in those aged 70 to 79 years had an $8.0 \%$ CFR and cases in those aged 80 years and older had a $14.8 \%$ CFR [7]. In some patients, severe pulmonary and extra-pulmonary complications may lead to respiratory failure and life-threatening events. It has been reported that about $50 \%$ of the patients had increased D-dimer levels, and abnormal D-dimer levels are associated with poor prognosis $[8,9]$. Thus, in some stable patients with sudden death, acute organ and embolism and infarction should take into consideration. Although the incidence of thrombosis in patients with COVID-19 has not been determined, the incidence of deep vein thrombosis (DVT) and pulmonary embolism (PE) was 20.5\% and $11.4 \%$ respectively in SARS cases [10]. In addition, thromboembolisms formation was seen in pathologic studies based on autopsies or biopsies, which greatly resemble those seen in SARS and MERS coronavirus infection [11, 12].

However, conventional anticoagulation may need to be considered carefully, as there is an increased risk of bleeding in patients with COVID-19 [13]. Therefore, in our experience, biomarkers, which can identify thrombus formation at earlier stages, might be used to evaluate the formation of thrombus and response to treatment. D-dimers are fibrin degradation products which have been shown to be useful in a clinical decision rule for ruling out pulmonary embolism [14], highlighting its role as a potentially helpful biomarker. However, the relationship between D-dimer and COVID-19 and the level changes during disease development were not fully reported. In this study, we compared the D-dimer levels of COVID19 patients with that of bacterial pneumonia, assessed the use of consecutive D-dimer levels after admission to hospital, and explored its association with markers of inflammation.

\section{Methods}

\section{Study design and participants}

This was a retrospective study done at two centres in China. The study was conducted in accordance with the principles of the Declaration of Helsinki and approved by the Medical Ethics Committee of the Second Xiangya Hospital of Central South University. The requirement for informed patient consent was waived by the Ethics Committee for this retrospective study that evaluated deidentified data involving no potential risk to patients and no link between the patients and the researchers. Patients with confirmed COVID-19 pneumonia who were admitted to Tongji Hospital of Tongji Medical College, and patients with confirmed community-acquired bacterial pneumonia in the Second Xiangya Hospital of Central South University, were retrospectively enrolled. Tongji Hospital is one of the designated hospitals for the hospitalization of patients with COVID-19 and one of its hospital wards has been entrusted by the Second Xiangya Hospital of Central South University since February 2020.

76 Patients with confirmed COVID-19 pneumonia enrolled in our department were screened. 57 Severe cases were included in our study (admission date from February 9 to February 15, 2020). Patients with secondary infection including bacteria and fungus, or patients lack of results of pre-treatment laboratory examination were excluded. The diagnosis of COVID-19 was determined with at least two positive results of real-time reverse transcriptase-polymerase chain reaction (RT-PCR) assay for SARS-CoV-2. Patients absent of or with negative SARS-CoV-2 test results were also excluded from this study. The COVID19 pneumonia has been classified to four types clinically by Guidelines for diagnosis and treatment of COVID-19 infection by the National Health Commission [15]. The classification of the four clinical types is as follow: (1) Mild: symptoms very mild, no pneumonia manifestation in CT; (2) Ordinary: fever, respiratory tract symptoms, and pneumonia manifestation in CT scan; (3) Severe: respiratory distress (respiratory rate $>30 / \mathrm{min}$ ), oxygen saturation $\leq 93 \%$ at rest, and $\mathrm{PaO}_{2} / \mathrm{FiO}_{2} \leq 300 \mathrm{mmHg}$; (4) Critical: respiratory failure need mechanic ventilation, shock, and combined multi-organs failure. All patients were clinically classified as severe at the time of admission. 46 Patients with laboratory-identified bacterial pneumonia by pathogenic detection were collected between August 1, 2019, and March 1, 2020. The Padua prediction score is a risk assessment model used to identify medical patients at high risk for venous thromboembolism (VTE). Demographic information, clinical characteristics including medical history, exposure history, comorbidities, signs, 
and symptoms, chest computed tomographic (CT) scan or X-ray results, and laboratory findings of each patient were obtained from the electronic medical record system of these two centres and analyzed by three independent researchers.

\section{Laboratory testing}

All medical laboratory data including the numbers of leukocytes, lymphocytes, and eosinophils; percentages of lymphocyte and eosinophils; concentrations of D-dimer, highsensitivity C-reactive protein (hsCRP), procalcitonin (PCT), and serum creatine kinase were generated by the clinical laboratory of Tongii Hospital and the Second Xiangya Hospital. The samples for laboratory tests were collected on admission and during the hospital stay. Peripheral venous blood was collected for routine blood test using automatic hematology analyzer. The biochemical parameters such as liver and renal function were measured by Roche automated clinical chemistry analyzer (Roche Diagnostics, Mannheim, Germany). Serum PCT was measured by the luminescence immunoassay, and hsCRP was measured with a latex particle-enhanced immunoturbidimetric assay (Roche Diagnostics). It is worth mentioning that coagulation tests were detected using a STA-R MAX coagulation analyzer and original reagents (Diagnostica Stago, Saint-Denis, France) in both hospitals. The laboratory data for some patients were missing due to the absence of types of tests or delayed results.

\section{Statistical analysis}

Continuous measurements were expressed as mean $\pm \mathrm{SD}$ if they are normally distributed or median (IQR) if they are not, and their differences were compared by the Student's t-test or Mann-Whitney U-test or Kruskal-Wallis test or Wilcoxon signed rank test. Besides, categorical variables were expressed as count (\%) and compared by $\chi^{2}$ test or Fisher's exact test. The relationship among biomarkers were assessed using Spearman's correlations analysis. We used SPSS (version 25.0) and Graph Pad Prism (version 7.0) for all analyses. Two-tailed $\mathrm{P}$ values $<0.05$ were considered statistically significant.

\section{Results}

\section{Baseline characteristics of the study population}

The study population included 57 hospitalized patients with COVID-19 and 46 hospitalized patients with community acquired pneumonia (CAP). For COVID-19 patients, the median age was 65 years (IQR 54-72), and $44 \%$ were men. For CAP patients, the median age was 64 years (IQR
Table 1 Baseline characteristics of patients on admission

\begin{tabular}{lllr}
\hline Variable & $\begin{array}{l}\text { COVID-19 } \\
\text { patients } \\
(\mathrm{n}=57)\end{array}$ & $\begin{array}{l}\text { CAP patients } \\
(\mathrm{n}=46)\end{array}$ & P value \\
\hline Age (years) & $65(54,72)$ & $64(60,70)$ & 0.471 \\
Sex & $25(44 \%)$ & $27(59 \%)$ & 0.134 \\
Men & $32(56 \%)$ & $19(41 \%)$ & \\
$\quad$ Women & $4(7 \%)$ & $10(22 \%)$ & 0.030 \\
Cardiovascular & & & $<0.001$ \\
$\quad$ diseases & $1(2 \%)$ & $16(35 \%)$ & 0.672 \\
Pulmonary disease & $18(39 \%)$ & 0.300 \\
Hypertension & $20(35 \%)$ & $11(24 \%)$ & 0.464 \\
Diabetes & $9(16 \%)$ & $3(7 \%)$ & $<0.001$ \\
Kidney diseases & $1(2 \%)$ & $22(48 \%)$ & 0.119 \\
Smoking & $1(2 \%)$ & $30(65 \%)$ & $<0.001$ \\
Fever & $45(79 \%)$ & $40(87 \%)$ & 0.123 \\
Cough & $24(42 \%)$ & $28(61 \%)$ & 0.786 \\
Shortness of breath & $26(46 \%)$ & $3(7 \%)$ & 0.654 \\
Myalgia & $3(5 \%)$ & $15(33 \%)$ & 0.879 \\
Chest distress & $21(37 \%)$ & $2(4 \%)$ & 0.097 \\
Diarrhoea & $4(7 \%)$ & $8(17 \%)$ & $<0.001$ \\
Inappetence & $3(5 \%)$ & $16(35 \%)$ & \\
Fatigue & $4(7 \%)$ & & \\
\hline & & & \\
\hline
\end{tabular}

Data are mean $\pm \mathrm{SD}$, or medians (25th-75th percentile) and $\mathrm{n}(\%)$

COVID-19 patients novel coronavirus pneumonia patients, CAP patients community acquired pneumonia

60-70), and 59\% were men (Table 1). Both of the COVID19 patients and CAP patients had 1 or more coexisting medical conditions, and compared with COVID-19 patients, CAP patients were more likely to have coexisting medical conditions, including cardiovascular disease (COVID-19 patients vs CAP patients: 4 [7\%] vs 10 [22\%]), pulmonary disease (1 [2\%] vs 16 [35\%]), and smoking (1 [2\%] vs 22 [48\%]) (Table 1).

On admission, no matter in COVID-19 patients or CAP patients, most patients had fever, cough, shortness of breath, myalgia, chest distress, diarrhea, inappetence and fatigue. Besides, there were numerous differences in laboratory findings (Table 2). Compared with COVID-19 patients, CAP patients were more likely to have higher white blood cell (WBC) and neutrophil counts (N), as well as higher procalcitonin (PCT), erythrocyte sedimentation rate (ESR) and fibrinogen (FIB), conversely, lower activated partial thromboplastin time (APTT), and there were no significant differences in other biomarkers levels between two groups.

\section{D-dimer levels were related with markers of inflammation}

To investigate whether D-dimer levels is associated with levels of inflammatory factors, we performed Spearman's 
Table 2 Laboratory results of patients with different pneumonia on admission

\begin{tabular}{|c|c|c|c|}
\hline Variable & COVID-19 patients $(\mathrm{n}=57)$ & CAP patients $(n=46)$ & $P$ value \\
\hline \multicolumn{4}{|l|}{ Blood routine } \\
\hline WBC $\left(\times 10^{9}\right.$ cells/L) (normal range 3.5-9.5) & $5.5(3.8,6.9)$ & $6.9(5.2,11.2)$ & 0.001 \\
\hline $\mathrm{N}\left(\times 10^{9}\right.$ cells $\left./ \mathrm{L}\right)($ normal range $1.8-6.3)$ & $3.4(2.1,4.6)$ & $5.3(3.7,9.4)$ & $<0.001$ \\
\hline $\mathrm{L}\left(\times 10^{9}\right.$ cells/L) (normal range $\left.1.1-3.2\right)$ & $1.3(0.8,1.6)$ & $1.0(0.7,1.7)$ & 0.318 \\
\hline PLT $\left(\times 10^{9}\right.$ cell/L) (normal range $\left.125.0-350.0\right)$ & $241.0(182.8,324.0)$ & $253.0(174.8,395.8)$ & 0.532 \\
\hline HGB $(\mathrm{g} / \mathrm{L})($ normal range $125.0-175.0)$ & $123.4 \pm 16.3$ & $112.4 \pm 19.0$ & 0.001 \\
\hline \multicolumn{4}{|l|}{ Coagulation function } \\
\hline D-dimer $(\mu \mathrm{g} / \mathrm{mL})$ (normal range $0.0-0.5)$ & $0.8(0.4,1.8)$ & $0.4(0.2,1.0)$ & $<0.01$ \\
\hline APTT (s) (normal range 29.0-42.0) & $39.5 \pm 6.2$ & $36.0 \pm 9.1$ & 0.033 \\
\hline PT (s) (normal range 11.5-14.5) & $13.8 \pm 1.1$ & $13.5 \pm 2.1$ & 0.123 \\
\hline TT (s) (normal range 14.0-19.0) & $17.0(15.9,18.0)$ & $16.7(16.0,17.5)$ & 0.378 \\
\hline INR (normal range $0.8-1.2$ ) & $1.1 \pm 1.0$ & $1.1 \pm 0.2$ & 0.108 \\
\hline FIB (g/L) (normal range 2.0-4.0) & $4.8 \pm 1.5$ & $5.6 \pm 2.0$ & 0.014 \\
\hline \multicolumn{4}{|l|}{ Blood biochemistry } \\
\hline Albumin (g/L) (normal range 35.0-52.0) & $34.0 \pm 3.9$ & $32.5 \pm 5.4$ & 0.118 \\
\hline Globulin (g/L) (normal range 20.0-35.0) & $34.5 \pm 5.4$ & $31.8 \pm 5.9$ & 0.011 \\
\hline $\mathrm{TP}(\mathrm{g} / \mathrm{L})$ (normal range 64.0-83.0) & $68.5 \pm 4.6$ & $64.1 \pm 7.5$ & 0.001 \\
\hline ALT (U/L) (normal range $0.0-41.0$ ) & $21.5(13.3,37.7)$ & $24.3(17.3,48.7)$ & 0.207 \\
\hline AST (U/L) (normal range $0.0-40.0$ ) & $26.5(18.8,34.5)$ & $29.1(21.7,40.5)$ & 0.135 \\
\hline BUN (mmol/L) (normal range 3.1-8.0) & $4.3(3.3,5.9)$ & $5.1(3.7,7.1)$ & 0.174 \\
\hline $\mathrm{Cr}(\mu \mathrm{mol} / \mathrm{L})($ normal range $59.0-104.0)$ & $67.0(57.0,82.0)$ & $67.2(52.3,80.8)$ & 0.840 \\
\hline CK (U/L) (normal range $0.0-170.0$ ) & $67.0(29.0,178.0)$ & $78.1(37.9,106.9)$ & 0.921 \\
\hline LDH (U/L) (normal range 135.0-225.0) & $288.8 \pm 104.7$ & $241.1 \pm 67.4$ & 0.048 \\
\hline Myoglobin (ng/mL) (normal range 0.0-154.9) & $31.4(22.4,53.5)$ & $64.9(42.3,92.8)$ & 0.004 \\
\hline \multicolumn{4}{|l|}{ Infection-related biomarkers } \\
\hline hsCRP $(\mathrm{mg} / \mathrm{L})$ (normal range $0.0-1.0)$ & $15.6(3.8,40.0)$ & $82.8(12.3,127.8)$ & $<0.001$ \\
\hline PCT $(\mathrm{ng} / \mathrm{mL})$ (normal range $0.02-0.05)$ & $0.04(0.02,0.09)$ & $0.1(0.05,0.42)$ & $<0.001$ \\
\hline ESR $(\mathrm{mm} / \mathrm{h})($ normal range $0.0-20.0)$ & $32.0(18.5,63.8)$ & $72.0(33.0,98.5)$ & 0.001 \\
\hline
\end{tabular}

Data are mean $\pm \mathrm{SD}$, or medians (25th-75th percentile)

COVID-19 patients novel coronavirus pneumonia patients, CAP patients community acquired pneumonia, $W B C$ white blood cell count, $N$ neutrophil count, $L$ lymphocyte count, $P L T$ platelet count, $H G B$ haemoglobin, $A P T T$ activated partial thromboplastin time, $P T$ prothrombin time, $T T$ thrombin time, INR international normalized ratio, FIB fibrinogen, $T P$ total protein, $A L T$ alanine aminotransferase, AST aspartate aminotransferase, $B U N$ blood urea nitrogen, $C r$ serum creatinine, $C K$ creatine kinase, $L D H$ lactate dehydrogenase, $h s C R P$ hypersensitive C-reactive protein, $P C T$ procalcitonin, ESR Erythrocyte sedimentation rate

correlations analysis between D-dimer levels and infectionrelated biomarkers levels in COVID-19 patients and CAP patients. As shown in Table 3, for COVID-19 patients, D-dimer levels were positively correlated with infectionrelated biomarkers levels including hsCRP, PCT and ESR before treatments $(\mathrm{R}=0.426,0.349,0.345$ respectively, $\mathrm{P}<0.05)$. And $\mathrm{D}$-dimer levels also had great correlations with inflammatory cells levels before treatments such as WBC, N, L $(\mathrm{R}=0.402,0.464,-0.426$, respectively, $\mathrm{P}<0.01)$ and coagulation function-related factors levels such as PT, INR $(\mathrm{R}=0.368,0.386$, respectively, $\mathrm{P}<0.01)$. In addition, for CAP patients, there were also positive correlations between D-dimer levels and infection-related biomarkers levels before treatments including hsCRP, PCT $(R=0.300,0.391$, respectively, $\mathrm{P}<0.05$, Table 4), and D-dimer levels were also related with other biomarkers levels before treatments like PT, APTT, INR and CK $(\mathrm{R}=0.374,0.383,0.398,-0.464$, respectively, $\mathrm{P}<0.05$, Table 4). At the same time, we also analyzed the correlations between these indicators after treatments in COVID-19 patients, and found that there were still great correlations between D-dimer and the same biomarkers as above, their correlation coefficients $\mathrm{R}>0.3(\mathrm{P}<0.05)$. However, due to the absence of following-up data, we couldn't analyze these relationships between post-treatment biomarkers levels in CAP patients.

More importantly, we found that in COVID-19 patients the correlation between D-dimer levels and hsCRP levels before treatments was related to the levels of hsCRP, while the levels of hsCRP exceed $10 \mathrm{mg} / \mathrm{L}$, the correlation between 
Table 3 Spearman's correlation coefficients between D-dimer and other biomarkers in COVID-19 patients

\begin{tabular}{lcc}
\hline D-dimer and & Untreated & Treated \\
\hline hsCRP & $0.426^{* *}$ & $0.495 * *$ \\
PCT & $0.349^{*}$ & $0.659 * *$ \\
ESR & $0.345^{*}$ & $0.511^{*}$ \\
WBC & $0.402 * *$ & 0.325 \\
N & $0.464 * * *$ & $0.462 * *$ \\
L & $-0.426 * *$ & $-0.400^{*}$ \\
PT & $0.368^{* *}$ & 0.234 \\
APTT & -0.056 & 0.123 \\
TT & -0.016 & 0.103 \\
INR & $0.386^{* *}$ & 0.194 \\
FIB & $0.282^{*}$ & $0.369 *$ \\
CK & 0.151 & -0.287 \\
\hline
\end{tabular}

The correlations between D-dimer and other biomarkers before(untreated) and after(treated) treatments in COVID-19 patients $* * * \mathrm{P}<0.001, * * \mathrm{P}<0.01, * \mathrm{P}<0.05$

Table 4 Spearman's correlation coefficients between D-dimer and other biomarkers in CAP patients

\begin{tabular}{lc}
\hline D-dimer and & Untreated \\
\hline hsCRP & $0.300^{*}$ \\
PCT & $0.391^{* *}$ \\
ESR & 0.273 \\
WBC & 0.038 \\
$\mathrm{~N}$ & 0.046 \\
$\mathrm{~L}$ & 0.006 \\
$\mathrm{PT}$ & $0.374^{*}$ \\
$\mathrm{APTT}$ & $0.383^{*}$ \\
$\mathrm{TT}$ & -0.083 \\
$\mathrm{INR}$ & $0.398^{* *}$ \\
FIB & 0.219 \\
$\mathrm{CK}$ & $-0.464^{* *}$ \\
\hline The correlations & between \\
$\mathrm{D}-$ dimer and other & biomark- \\
ers before(untreated) & in CAP \\
patients & \\
$* * * \mathrm{P}<0.001$, & $* * \mathrm{P}<0.01$, \\
$* \mathrm{P}<0.05 \quad$ &
\end{tabular}

D-dimer and hsCRP was stronger (hsCRP $<10 \mathrm{mg} / \mathrm{L}$ vs hsCRP $\geq 10 \mathrm{mg} / \mathrm{L}, \mathrm{R}=-0.212$ vs 0.448 , Table 5 ).

\section{D-dimer levels were higher in COVID-19 patients compared with CAP patients on admission}

To explore the difference of $\mathrm{D}$-dimer levels between COVID-19 patients and CAP patients, we divided the levels of untreated hsCRP into two groups both in COVID19 patients and CAP patients, one group for hsCRP levels $<30 \mathrm{mg} / \mathrm{L}$, and another group for hsCRP $\geq 30 \mathrm{mg} / \mathrm{L}$,
Table 5 Spearman's correlation coefficients between D-dimer and related biomarkers according to untreated hsCRP levels in COVID-19 patients

\begin{tabular}{lll}
\hline D-dimer and & hsCRP $<10 \mathrm{mg} / \mathrm{L}$ & hsCRP $\geq 10 \mathrm{mg} / \mathrm{L}$ \\
\hline hsCRP & & \\
Untreated & -0.212 & $0.448^{* *}$ \\
Treated & -0.268 & 0.348 \\
PCT & & \\
Untreated & -0.178 & 0.320 \\
Treated & $<0.00$ & 0.449 \\
CK & & \\
Untreated & 0.371 & -0.067 \\
Treated & - & -0.304 \\
\hline
\end{tabular}

$* * * \mathrm{P}<0.001, * * \mathrm{P}<0.01, * \mathrm{P}<0.05$

according to the median hsCRP level in 103 patients. We found that no matter in COVID-19 patients or CAP patients, the higher hsCRP levels, the higher D-dimer levels (Figs. 1a, 2a). Besides, this trend also existed in other biomarkers levels, including PCT, FIB and INR (Figs. 1b-f, 2b-f). As shown in Table 2, hsCRP levels were 15.6 (3.8-40.0) mg/L in COVID-19 patients, and 82.8 (12.3-127.8) $\mathrm{mg} / \mathrm{L}$ in CAP patients, thus, we also grouped COVID-19 patients or CAP patients at their median level of hsCRP respectively, and found that all the trends remained unchanged (Figs. S2, S3).

Interestingly, it was worth mentioning that compared with COVID-19 patients, the levels of hsCRP were higher in CAP patients, whereas the levels of D-dimer were lower in CAP patients (Fig. 3a, b).

\section{In COVID-19 patients with good clinical prognosis, hsCRP levels decreased after treatment, while D-dimer levels decreased synchronously}

As previous described, D-dimer levels were truly related with biomarkers of inflammation, especially with hsCRP. We then analyzed the specific relationship between D-dimer levels and hsCRP levels in COVID-19 patients, and found that both hsCRP levels and D-dimer levels decreased after treatments (Fig. 4a, b). Moreover, we analyzed their relationship before and after treatments stratified by untreated hsCRP quartiles, as expected, after therapy, hsCRP levels were significantly decreased in the $2 \mathrm{nd}$, 3rd and 4th quartiles of untreated hsCRP (Fig. S1a-d), and there were also a downward trend in D-dimer levels at different quartiles (Fig. S1e-h).

However, considering that the values stratified by untreated hsCRP quartiles might be higher or lower cutoff values which could bias the results, and as previously described, hsCRP levels were significantly decreased in the 2nd quartile, we then divided all patients into two groups 

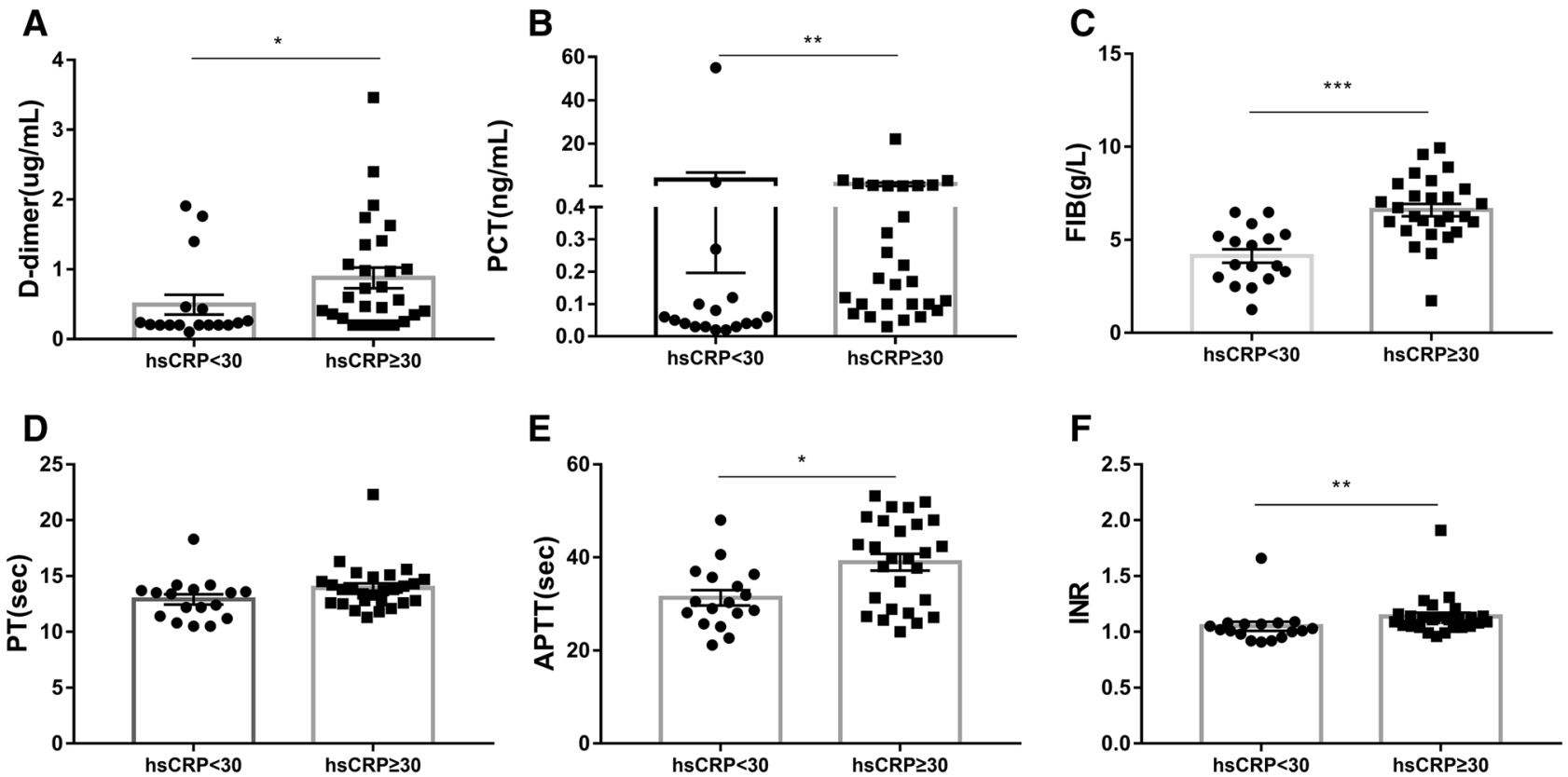

Fig. 1 On admission, changes in biomarkers levels based on different levels of hsCRP in CAP patients. The hsCRP levels were divided into

a D-dimer, b PCT, c FIB, d PT, e APTT and f INR. ***P $<0.001$, two groups, $h s C R P<30 \mathrm{mg} / \mathrm{L}$ group, and $\mathrm{hsCRP} \geq 30 \mathrm{mg} / \mathrm{L}$ group.

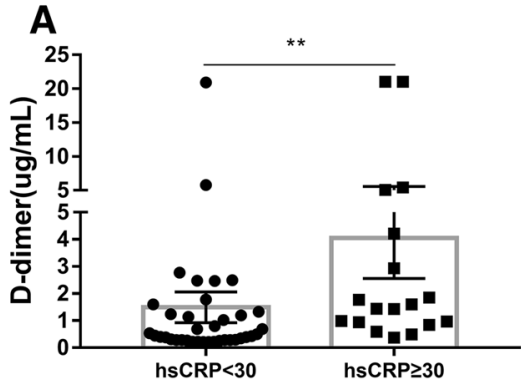

D

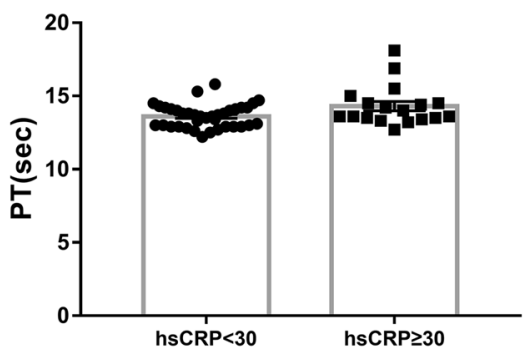

B

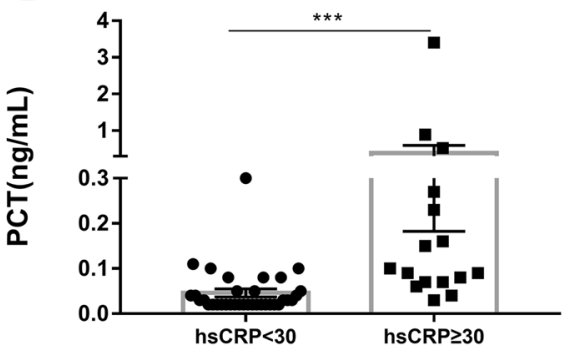

E

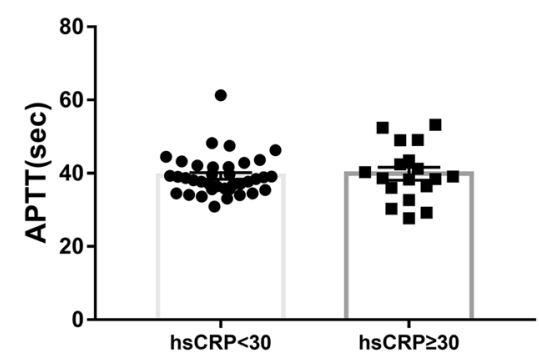

C

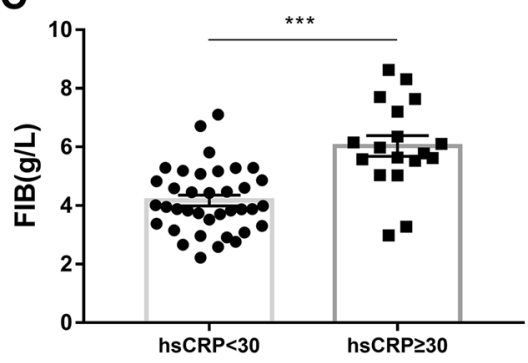

$\mathbf{F}$

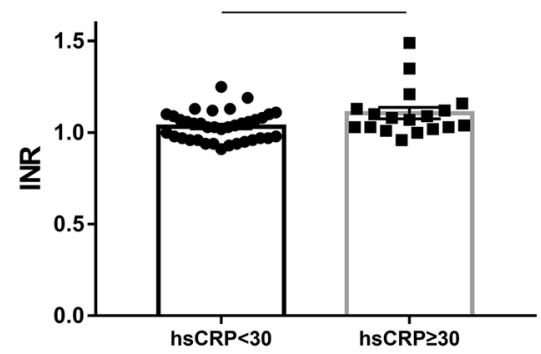

Fig. 2 On admission, changes in biomarkers levels based on different levels of hsCRP in COVID-19 patients. The hsCRP levels were divided into two groups, $\mathrm{hsCRP}<30 \mathrm{mg} / \mathrm{L}$ group, and
$\mathrm{hsCRP} \geq 30 \mathrm{mg} / \mathrm{L}$ group. a D-dimer, b PCT, c FIB, d PT, e APTT and $\mathbf{f}$ INR. $* * * \mathrm{P}<0.001, * * \mathrm{P}<0.01, * \mathrm{P}<0.05$

In addition, it's worth mentioning that there were 53 patients were cured or turned into mild cases, whereas 4 patients were died in our study. More important, we found that in deceased patients, both the untreated hsCRP or 
Fig. 3 On admission, comparison between the levels of D-dimer and hsCRP in COVID-19 patients and CAP patients. a Changes in levels of D-dimer between two groups and $\mathbf{b}$ changes in levels of hsCRP between two groups. $* * * \mathrm{P}<0.001, * * \mathrm{P}<0.01$ $* \mathrm{P}<0.05$
A

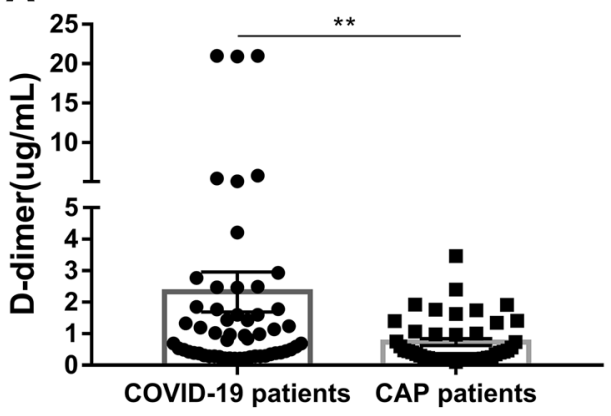

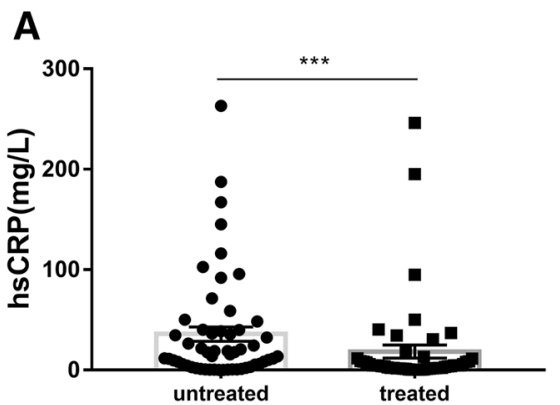

B

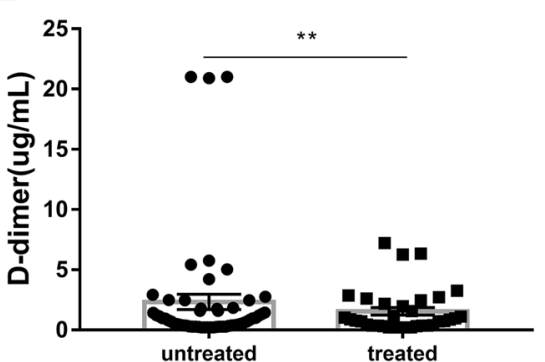

E

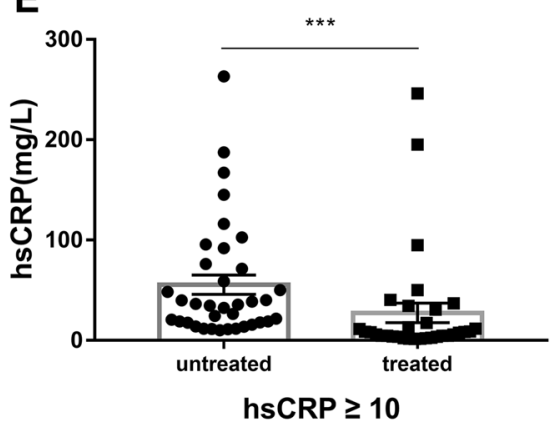

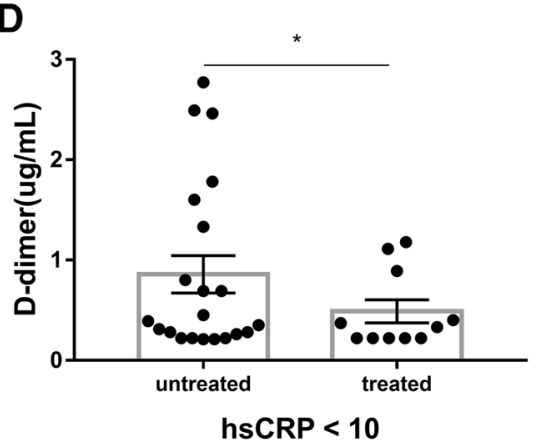

C
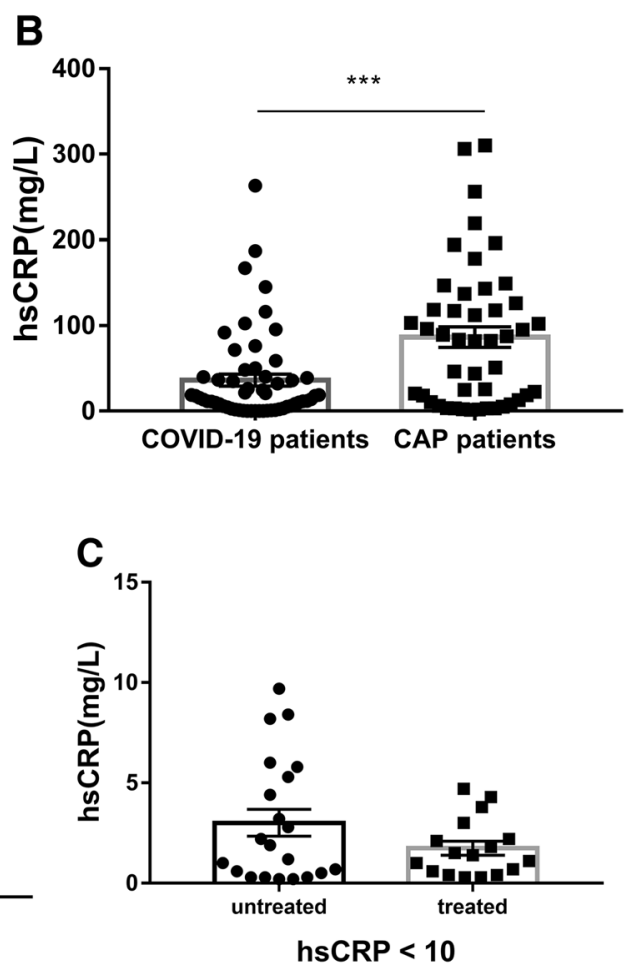

$\mathbf{F}$

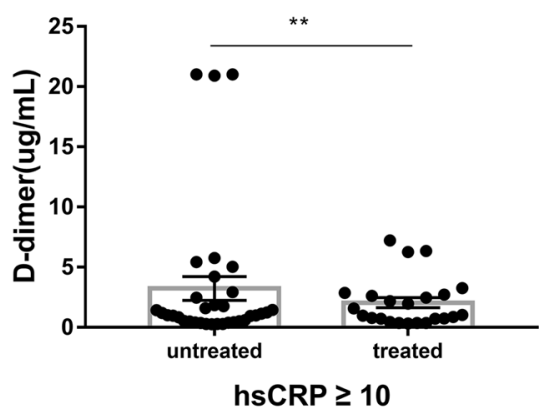

Fig. 4 Changes in hsCRP and D-dimer levels after treatments in COVID-19 patients. Changes in hsCRP and D-dimer levels after treatments in all patients, a hsCRP levels, b D-dimer levels. Changes in hsCRP and D-dimer levels after treatments at different untreated
hsCRP levels, and the untreated hsCRP levels were divided into two groups: hsCRP $<10 \mathrm{mg} / \mathrm{L}$ group; hsCRP $\geq 10 \mathrm{mg} / \mathrm{L}$ group. $\mathbf{c}$ hsCRP, d D-dimer, while hsCRP $<10 \mathrm{mg} / \mathrm{L}$, e hsCRP, and f D-dimer, while hsCRP $\geq 10 \mathrm{mg} / \mathrm{L}$. $* * * \mathrm{P}<0.001, * * \mathrm{P}<0.01, * \mathrm{P}<0.05$
D-dimer levels and treated hsCRP or D-dimer levels were still abnormally high (Table S1), conversely, both hsCRP and D-dimer levels significantly decreased in patients with a good clinical prognosis after therapy.

\section{In COVID-19 patients, some patients had a significant decrease in hsCRP levels after therapy, whereas D-dimer levels were increased}

As we know, D-dimer is one of the markers for thrombosis. However, the synchronous decline of D-dimer and hsCRP suggests that the elevated D-dimer levels in COVID-19 patients is related to inflammation, which limits its role in the prediction of thrombosis. Further analysis showing low correlation between Padua VTE score and D-dimer levels (Spearman's $\mathrm{R}=0.264, \mathrm{P}>0.05$ ) weakened the role of D-dimer in the prediction of thrombosis. Subsequently, in order to investigate whether the levels of D-dimer also decreased significantly in those patients with a significant decrease in hsCRP levels, we then analyzed the relationship between the extent of decline in hsCRP and D-dimer levels after treatments. Interestingly, it was worth mentioning that some patients had a significant decrease in hsCRP levels, whereas their D-dimer levels were increased (Fig. 5), highlighting the possibility for aggressive coagulation therapy. Therefore, for these patients, the anticoagulant therapy was strengthened, and the low molecular weight heparin was changed from the preventive dose to the therapeutic dose. 
Fig. 5 Changes in D-dimer and hsCRP after treatments at two groups in COVID-19 patients, and the difference value $=$ untreated hsCRP levels minus treated hsCRP levels, the two groups were divided into the difference value $<5 \mathrm{mg} / \mathrm{L}$ and the difference value $\geq 5 \mathrm{mg} / \mathrm{L}$. a, b Changes in levels of hsCRP after treatments and $\mathbf{c}, \mathbf{d}$ changes in levels of D-dimer after treatments

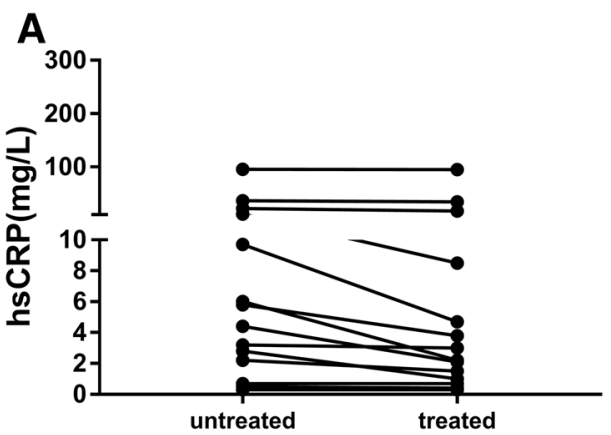

Difference value $<5$

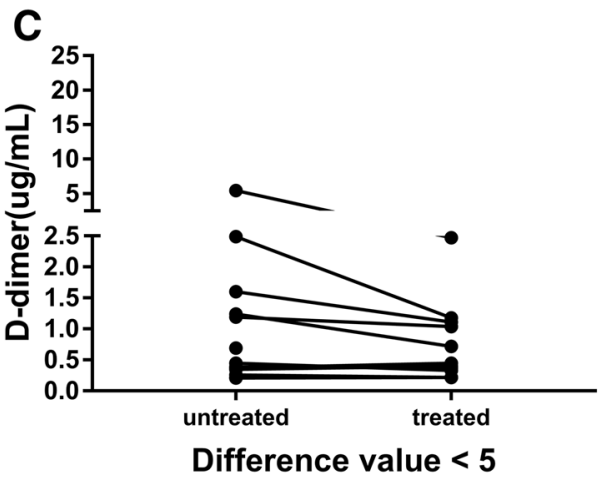

B

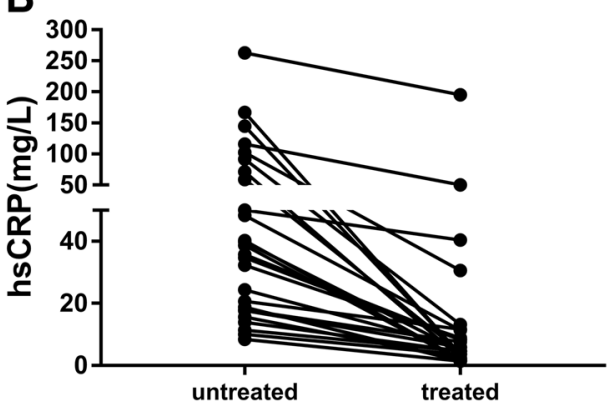

Difference value $\geq 5$

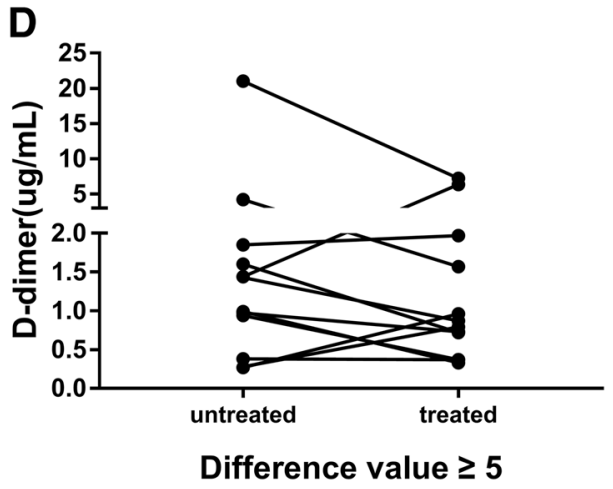

\section{Discussion}

In this analysis of our enrolled patients with COVID-19 and bacterial pneumonia, we demonstrated that COVID19 patients with significantly higher D-dimer levels, which highlighting the possibility of more obvious activation of the coagulation system. Although D-dimer levels correlated with inflammatory markers and tended to normalize in convalescent stage in most of the patients, the anomalous rise might be an indicator of active anticoagulant therapy. Although it has been reported that D-dimer is significantly increased in patients with COVID-19 and is related to prognosis [16, 17], this is the first report about the relationship between D-dimer levels and the markers of inflammation in COVID-19 as well as the variation during disease progression.

Elevated D-dimer levels have been reported in a limited number of studies involved in both SARS and CAP patients [18-21]. As demonstrated in our study, similar to SARS and CAP patients, the D-dimer levels of COVID-19 patients was also elevated. The reasons responsible for the elevated D-dimer levels are only partially explained. It is well known that D-dimer are produced during fibrin breakdown and serve as a marker of fibrinolytic activity. A relationship between proinflammatory cytokines and markers of activation of the coagulation cascade, including D-dimer, has been demonstrated in critical patients or patients with sepsis $[22,23]$. There is also evidence that under inflammatory conditions, the alveolar haemostatic balance is shifted towards a predominance of prothrombotic activity [24]. In addition, pro-inflammatory cytokines may be involved in endothelial injury, and may activate coagulation and inhibit fibrinolysis in patients with severe sepsis [25]. In this paper, the relationship between D-dimer levels and the markers of inflammation were also analysed in both COVID and CAP patients. Although we lost the post-treatment data for CAP patients, all the data reported in our analysis showed that D-dimer levels were significantly correlated with inflammation and tended to normalize as the inflammation subsided in most of the patients, highlighting the point that inflammation is one of the causes of coagulation activation in patients with both COVID and bacterial pneumonia.

However, one problem that could not be ignored is that patients with COVID-19 have higher levels of D-dimer when their CRP levels are lower than that of CAP patients. This highly suggests that there are other factors besides inflammation that responsible for activation of the coagulation system in patients with COVID-19. In a previous study [26], Gralinski et al. investigated viral pathogenesis and identify a novel host pathway involved in SARS progression. Their data suggest that dysregulation of the urokinase pathway during SARS-coronavirus infection contributes to more severe lung pathology and profound alterations in the systemic haemostatic balance.

In the treatment of patients with COVID-19, the prevention and treatment of thrombus should be noted. It has been reported that reactive thrombocytosis occurred in $4 \%$ 
of patients, which may be related to the increased risk of thrombus [8]. In addition, considering that patients with COVID-19 might have increased blood viscosity due to high fever and excessive sweating, hypercoagulable state because of activation of coagulation system [9], together with the risk factors such as long-term bedridden, obesity and old age, the risk of thrombus is further increased. The diagnostic value of $\mathrm{D}$-dimer levels for thrombus formation in COVID-19 patients is unclear. Whether higher D-dimer levels in patients with COVID-19 suggest the need for more aggressive anticoagulant therapy deserves further discussion. Previous studies demonstrated high D-dimer levels in patients with CAP similar to patients with pulmonary embolism that decreased the reliability of the test for the differential diagnosis of CAP and pulmonary embolism [19]. As shown in our study, levels of D-dimer decreased with the disappearance of inflammation and the improvement of the disease, which indicates that it is not reasonable to judge whether anticoagulation is needed only according to D-dimer levels. Also, the low correlation between Padua VTE score and D-dimer levels weakened the role of D-dimer in the prediction of thrombosis. This raises the question of what indicators are used to guide prophylaxis for venous thromboembolism in patients with COVID-19. Interestingly, not all patients' D-dimer levels decreased with the decrease of inflammatory factors, suggesting the possibility of thrombosis. Thus, it should be noted that if levels of D-dimer are not synchronized with the regression of inflammation, anticoagulation therapy is needed. In addition, VTE risk assessment should also be considered in clinical decision-making. Because the mortality of COVID-19 increases with age, and elderly patients are more likely to have bleeding events, bleeding scores should also be included in clinical decisions.

This study has some several limitations. It's a retrospective analysis and patients we included in the present analysis were not systematically assessed for the presence of pulmonary embolism and VTE because of conditional restriction. Furthermore, although the hsCRP and D-dimer levels were examined by the same method, the COVID-19 patients and CAP patients were enrolled in different centres, which might reduce the credibility of research results.

\section{Conclusion}

Elevated baseline D-dimer levels are associated with inflammation in COVID-19 patients and have limited predictive value for thrombosis. In the treatment of COVID-19 patients, the change of D-dimer levels should be observed dynamically. And the abnormal changes of D-dimer and inflammatory factors suggest that anticoagulant therapy might be needed. Also, although the predictive value of VTE score need to be further studied in COVID-19 patients, it might be useful than baseline D-dimer levels for prophylaxis for venous thromboembolism in COVID-19 patients.

Acknowledgements The authors sincerely appreciate all the Members of Medical Support Group from the Second Xiangya Hospital, Central South University to Tongji Hospital in Wuhan. Especially in memory of those doctors who devoted their life in this event to fight the COVID-19 pneumonia in China. Thanks to the Electronic Medical Record Systems of Tongji Hospital of Tongji Medical College and Second Xiangya Hospital for providing patients' data.

Author contributions Y-BL, XL, L-XL, T-JJ, Y-LZ, and H-GX contributed to the study design. HZ, Z-XG, LT, JC, O-MQ, QL, and MX contributed to data collection. HZ, JC and O-MQ did the data analyses. Y-BL, XL, JC, Y-LZ, L-XL, and T-JJ wrote the manuscript. All authors read and approved the final manuscript.

Funding Not applicable.

Data availability The datasets collected during the study are available from the corresponding author on reasonable request.

\section{Compliance with ethical standards}

Conflict of interest The authors declare that they have no competing interests.

Ethical approval The study was conducted in accordance with the principles of the Declaration of Helsinki and approved by the Medical Ethics Committee of the Second Xiangya Hospital of Central South University.

Informed consent The requirement for informed patient consent was waived by the Ethics Committee for this retrospective study that evaluated de-identified data involving no potential risk to patients and no link between the patients and the researchers.

\section{References}

1. Chen N, Zhou M, Dong X et al (2020) Epidemiological and clinical characteristics of 99 cases of 2019 novel coronavirus pneumonia in Wuhan, China: a descriptive study. Lancet 395(10223):507-513

2. Huang C, Wang Y, Li X et al (2020) Clinical features of patients infected with 2019 novel coronavirus in Wuhan, China. Lancet 395(10223):497-506

3. Wang D, Hu B, Hu C et al (2020) Clinical characteristics of 138 hospitalized patients with 2019 novel coronavirus-infected pneumonia in Wuhan, China. JAMA 323(11):1061-1069

4. Tan W, Zhao X, Ma X, Wang W, Niu P, Xu W, Gao GF, Wu $G$ (2020) A novel coronavirus genome identified in a cluster of pneumonia cases-Wuhan, China 2019-2020. China CDC Wkly 2(4):61-62

5. Tsang KW, Ho PL, Ooi GC et al (2003) A cluster of cases of severe acute respiratory syndrome in Hong Kong. N Engl J Med 348(20):1977-1985

6. Assiri A, Al-Tawfiq JA, Al-Rabeeah AA et al (2013) Epidemiological, demographic, and clinical characteristics of 47 cases of Middle East respiratory syndrome coronavirus disease from Saudi Arabia: a descriptive study. Lancet Infect Dis 13(9):752-761 
7. Wu Z, McGoogan JM (2020) Characteristics of and important lessons from the coronavirus disease 2019 (COVID-19) outbreak in China: summary of a report of 72314 cases from the Chinese Center for Disease Control and Prevention. JAMA. https://doi. org/10.1001/jama.2020.2648

8. Guan W, Ni Z, Hu Y et al (2020) Clinical characteristics of 2019 novel coronavirus infection in China. medRxiv. https://doi. org/10.1101/2020.02.06.20020974

9. Tang N, Li D, Wang X, Sun Z (2020) Abnormal coagulation parameters are associated with poor prognosis in patients with novel coronavirus pneumonia. J Thromb Haemost 18(4):844-847

10. Chong PY, Chui P, Ling AE et al (2004) Analysis of deaths during the severe acute respiratory syndrome (SARS) epidemic in Singapore: challenges in determining a SARS diagnosis. Arch Pathol Lab Med 128(2):195-204

11. Xu Z, Shi L, Wang Y et al (2020) Pathological findings of COVID-19 associated with acute respiratory distress syndrome. Lancet Respir Med 8(4):420-422

12. Lang Z, Zhang L, Zhang S, Meng X, Li J, Song C, Sun L, Zhou Y (2003) Pathological study on severe acute respiratory syndrome. Chin Med J Peking 07:17-21

13. Mao L, Wang M, Chen S et al (2020) Neurological manifestations of hospitalized patients with COVID-19 in Wuhan, China: a retrospective case series study. medRxiv. https://doi. org/10.1101/2020.02.22.20026500

14. van Belle A, Buller HR, Huisman MV et al (2006) Effectiveness of managing suspected pulmonary embolism using an algorithm combining clinical probability, D-dimer testing, and computed tomography. JAMA 295(2):172-179

15. Lin L, Li TS (2020) Interpretation of "Guidelines for the Diagnosis and Treatment of Novel Coronavirus (2019-nCoV) Infection by the National Health Commission (Trial Version 5)". Zhonghua Yi Xue Za Zhi 100:E001

16. Yin S, Huang M, Li D, Tang N (2020) Difference of coagulation features between severe pneumonia induced by SARS-CoV2 and non-SARS-CoV2. J Thromb Thrombolysis. https://doi. org/10.1007/s11239-020-02105-8

17. Zhang L, Yan X, Fan Q, Liu H, Liu X, Liu Z, Zhang Z (2020) D-dimer levels on admission to predict in-hospital mortality in patients with COVID-19. J Thromb Haemost. https://doi. org/10.1111/jth. 14859
18. Hui DS, Wong PC, Wang C (2003) SARS: clinical features and diagnosis. Respirology 8(Suppl):S20-S24

19. Snijders D, Schoorl M, Schoorl M, Bartels PC, van der Werf TS, Boersma WG (2012) D-dimer levels in assessing severity and clinical outcome in patients with community-acquired pneumonia. A secondary analysis of a randomised clinical trial. Eur J Intern Med 23(5):436-441

20. Salluh J, Rabello L, Rosolem MM, Soares M, Bozza FA, Verdeal J, Mello GW, Castro FNH, Lapa ESJ, Bozza PT (2011) The impact of coagulation parameters on the outcomes of patients with severe community-acquired pneumonia requiring intensive care unit admission. J Crit Care 26(5):496-501

21. Agapakis DI, Tsantilas D, Psarris P, Massa EV, Kotsaftis P, Tziomalos K, Hatzitolios AI (2010) Coagulation and inflammation biomarkers may help predict the severity of community-acquired pneumonia. Respirology 15(5):796-803

22. Shorr AF, Thomas SJ, Alkins SA, Fitzpatrick TM, Ling GS (2002) D-dimer correlates with proinflammatory cytokine levels and outcomes in critically ill patients. Chest 121(4):1262-1268

23. Pettila V, Hynninen M, Takkunen O, Kuusela P, Valtonen M (2002) Predictive value of procalcitonin and interleukin 6 in critically ill patients with suspected sepsis. Intensive Care Med 28(9):1220-1225

24. Gunther A, Mosavi P, Heinemann S, Ruppert C, Muth H, Markart P, Grimminger F, Walmrath D, Temmesfeld-Wollbruck B, Seeger W (2000) Alveolar fibrin formation caused by enhanced procoagulant and depressed fibrinolytic capacities in severe pneumonia. Comparison with the acute respiratory distress syndrome. Am J Respir Crit Care Med 161(2 Pt 1):454-462

25. Bone RC, Grodzin CJ, Balk RA (1997) Sepsis: a new hypothesis for pathogenesis of the disease process. Chest 112(1):235-243

26. Gralinski LE, Bankhead AR, Jeng S et al (2013) Mechanisms of severe acute respiratory syndrome coronavirus-induced acute lung injury. Mbio 4(4):e00271-e313

Publisher's Note Springer Nature remains neutral with regard to jurisdictional claims in published maps and institutional affiliations.

\section{Affiliations}

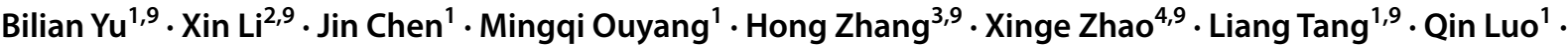 Min Xu ${ }^{5,9} \cdot$ Lizhen Yang ${ }^{6,9}$. Guxiang Huang ${ }^{7,9} \cdot$ Xianling Liu ${ }^{8,9}$. Jianjun Tang ${ }^{1,9}$}

1 Department of Cardiovascular Medicine, The Second Xiangya Hospital, Central South University, Changsha 410011, Hunan, China

2 Department of Vascular Surgery, The Second Xiangya Hospital, Central South University, Changsha 410011, Hunan, China

3 Department of Cardiovascular Surgery, The Second Xiangya Hospital, Central South University, Changsha 410011, Hunan, China

4 Department of Spine Surgery, The Second Xiangya Hospital, Central South University, Changsha 410011, Hunan, China

5 Department of Intensive Care Unit, The Second Xiangya Hospital, Central South University, Changsha 410011, Hunan, China
6 Department of Respiratory Intensive Care Unit, The Second Xiangya Hospital, Central South University, Changsha 410011, Hunan, China

7 Department of Geratology, Secondary Xiangya Hospital, Central South University, Changsha 410011, Hunan, China

8 Department of Oncology, Secondary Xiangya Hospital, Central South University, Changsha 410011, Hunan, China

9 Anti-2019-nCov Pneumonia Medical Support Group to Wuhan from the Second Xiangya Hospital, Central South University at Tongji Hospital of Tongji Medical College, Wuhan, Hubei, China 